\title{
Inflammatory bowel disease and laterality: is left handedness a risk?
}

\author{
D L Morris, S M Montgomery, M L Galloway, R E Pounder, A J Wakefield
}

\begin{abstract}
Background-Left handedness has been associated with inflammatory bowel disease (IBD) and autoimmune diseases. Aims-To determine whether left handedness is associated with IBD in two prospective national birth cohorts.

Methods-Subjects with Crohn's disease (CD) and ulcerative colitis (UC) were identified from two national longitudinal birth cohorts at age 26 years $(1970$ British Cohort Study (BCS70), born in 1970) and age 33 years (National Child Development Study (NCDS), born in 1958). Laterality was determined at age 10 (BCS70) or seven (NCDS) years, based on hand preference for writing and foot preference for kicking a ball (BCS70 only). Multiple logistic regression was used to assess the relationship of handedness with $\mathrm{CD}, \mathrm{UC}$, and IBD in the cohorts combined and adjusted for sex.
\end{abstract}

Results-Both cohorts combined showed increased adjusted relative odds of 2.13 (95\% confidence interval (CI) 0.97-4.65; $\mathrm{p}=0.059), 2.13(95 \%$ CI $0.92-4.91 ; \mathrm{p}=0$. $077)$, and 2.13 (95\% CI $1.20-3.78 ; p=0.010)$ for $\mathrm{CD}$, UC, and IBD, respectively in left handers.

Conclusions-The study suggests a link between IBD and left handedness which may be genetic and/or environmental in origin.

(Gut 2001;49:199-202)

Keywords: inflammatory bowel disease; handedness

Royal Free and University College Hospital Medical School, London, UK D L Morris

M L Galloway

R E Pounder

A J Wakefield

Enheten für Klinisk Epidemiologi,

Karolinska Sjukhuset and Institutet,

Stockholm, Sweden

S M Montgomery

Correspondence to:

Dr D L Morris,

Inflammatory Bowel Disease

Study Group, Royal Free and

University College Hospital

Medical School, Rowland

Hill Street, London

NW3 2PF, UK

Wisemail@compuserve.com

Accepted for publication

12 February 2000
Left handedness has been associated with various diseases, many of which are thought to have an autoimmune origin. These include asthma and other atopic conditions, migraine, thyroid disease, type I diabetes mellitus, autism, developmental learning disorders, and acquired immunodeficiency syndrome..$^{1-5}$

The inflammatory bowel diseases (IBDs) Crohn's disease (CD) and ulcerative colitis (UC) have also been linked with left handedness in some but not all studies. Geschwind and Behan originally described an association in 1982 based on self reporting of immune disorders in customers using a specialised shop for left handers. ${ }^{4}$ A case control study later found a significant increased odds ratio (OR) for left handedness in those with CD (OR 2.5, 95\% confidence interval (CI) 1.5-4.2), UC (OR $2.9,95 \%$ CI 1.4-5.9), and IBDs overall (OR $2.7,95 \%$ CI 1.7-4.4) after adjusting for sex. ${ }^{6}$ However, a hospital based case control study of 83 subjects with CD or UC failed to find such an association.
A meta-analysis of these studies supported a significant association between left handedness and IBD overall (OR 2.01, 95\% CI 1.352.98). ${ }^{8}$ Other studies have included both $\mathrm{CD}$ and UC with other diseases of "immune disorders" but have had insufficient numbers of patients to analyse them separately. ${ }^{9}{ }^{10}$

We have used prospectively collected data from two national population based birth cohorts to examine the risk of IBD in relation to hand preference. When combined, these form the largest and only nationally representative study in which the association between handedness and IBD has been examined. The study design therefore had the advantage of reducing many of the potential biases associated with previous case control studies.

\section{Subjects and measures} SUBJECTS

This study used data from two nationally representative birth cohort studies: the 1970 British Cohort Study (BCS70) and the National Child Development Study (NCDS). These prospective ongoing studies follow all those born during one week (5-11 April 1970 for the BCS70 and 3-9 March 1958 for the NCDS) who live in Great Britain. ${ }^{11}$

For the BCS70, a postal self completion survey was conducted in 1995-6 when cohort members were aged 25-26 years. This asked if they had "ever had or been told by a doctor that you had a diagnosis of Crohn's disease, ulcerative colitis". Data collected from an earlier BCS70 survey at 16 years, in addition, identified two cohort members with IBD. All self reported cases of IBD were contacted again by post to confirm their responses and to seek permission to contact their physician to confirm the diagnosis. ${ }^{12}$

The response rate of those subjects traced at age 26 years in the BCS70 cohort was $77 \% .^{12}$ Of 8150 subjects from the BCS70 used in these analyses, 21 had CD and 19 had UC.

For the NCDS, the diagnosis of IBD was established using the international coding of disease codes reported at face to face interviews when subjects were 23 or 33 years old. The response rate of those traced at 33 years was $85 \% .^{13}$ Physicians were not asked to confirm these diagnoses. Of 9771 subjects from the NCDS used in these analyses, 17 had CD and 16 had UC.

Abbreviations used in this paper: IBD,

inflammatory bowel disease; CD, Crohn's disease; UC, ulcerative colitis; OR, odds ratio; BCS, British Cohort Study; NCDS, National Child Development Study. 
Table 1 Relative odds for the association between left handedness and sex in two national birth cohorts

\begin{tabular}{llll}
\hline Sex & $\begin{array}{l}\text { Left handed subjects } \\
(\%)\end{array}$ & $\begin{array}{l}\text { Relative odds } \\
(95 \% \text { CI })\end{array}$ & $\begin{array}{l}\text { Pearson's two } \\
\text { tailed } p \text { value }\end{array}$ \\
\hline Male & $1045 / 8379(12.5 \%)$ & $1.28(1.17-1.41)$ & 0.000 \\
Female & $882 / 8818(10.0 \%)$ & 1.00 & \\
\hline
\end{tabular}

Despite loss to follow up in both cohorts since their inception, cohort members for whom data were available were broadly representative of the original cohorts, with approximately two thirds of both cohorts used in the analyses having a father with a manual social class. ${ }^{1415}$

MEASURES

Midwives recorded the sex of each subject at birth in 1958 (NCDS) or 1970 (BCS70).

For the BCS70, handedness and foot preference were ascribed following a medical examination and interview at age 10 years. The child was asked: "which hand is used for writing?" and "which foot is used for kicking a ball?" Responses of right, left, either, or unknown were recorded.

For the NCDS, the response of parents at interview with a health visitor was recorded in 1965 when the children were seven years old. The question "does the mother think the child is: right handed, left handed, mixed right and left, or don't know" was used.

"Responses of "either" in the BCS70 or "mixed right and left" in the NCDS were excluded from the analysis.

STATISTICAL ANALYSIS

The two cohorts were combined to increase statistical power. Cross tabulation was used to investigate the relationship between sex and IBD and sex and handedness, as sex was considered to be a potential confounding factor. Adjustment was similarly made for cohort.

Cross tabulation and Pearson's $\chi^{2}$ test were used to assess the relationships between handedness and UC, CD, and IBD. These were performed after combining both cohorts. Due to the small numbers, the Fisher's exact test was used to assess foot preference to kick a ball in the BCS70. Relative odds were calculated with $95 \%$ CI. All $\mathrm{p}$ values reported were two tailed. Logistic regression analysis was used to adjust for sex and birth cohort.

\section{Results}

Complete data on handedness and sex were available for $8134 / 9757$ (83\%) subjects from the BCS70 who responded at age 26 years and from $9062 / 11184(81 \%)$ subjects from the NCDS who responded at age 33 years. Overall,
71 patients with complete data available for analysis were identified with IBD (38 with CD and 33 with UC).

For foot preference, data from 7691/9757 $(78 \%)$ and $8134 / 9757$ (83\%) subjects from the BCS70, respectively, were available for analysis (20 with CD, 18 with UC).

\section{HANDEDNESS AND SEX}

Sex was considered to be a potential confounding factor even though there was no statistically significant association between $\mathrm{CD}, \mathrm{UC}$, or IBD combined and sex in this study. Left handedness showed a statistically significant association with male sex in both cohorts combined $\left(\chi^{2}=26.3, p=0.000\right)($ table 1$)$.

\section{COHORT AND IBD}

There were 31 patients with IBD in the NCDS and 40 in the BCS70 who had complete data available for analysis. A diagnosis of IBD was more likely in the BCS70 cohort than in the NCDS (unadjusted OR 1.44, 95\% CI 0.92.37; $\mathrm{p}=0.109)$.

HANDEDNESS AND IBD

Both cohorts showed increased relative odds for IBD in left handers. These were statistically significant for the BCS70 (adjusted for sex, OR 2.28, 95\% CI 1.08-4.79; Fisher's $p=0.031$ ) but not for the NCDS (adjusted for sex, OR 1.94, 95\% CI 0.79-4.75; Fisher's p=0.147).

Left handers had increased relative odds for both CD and UC when analysed as separate diseases in the combined cohorts although this was not statistically significant. Adjusting for sex and cohort did not alter the significance of the association (table 2)

IBD (CD or UC) was significantly associated with left handedness when the two cohorts were combined. Again, adjusting for sex and cohort did not alter these findings significantly (table 2).

FOOT PREFERENCE AND IBD

Data on foot preference were only available for the BCS70 cohort. There was no statistically significant association between foot preference and CD. For those with UC, $27.8 \%$ showed left foot preference compared with $12.7 \%$ of those without the disease (unadjusted relative odds 2.64, 95\% CI 0.94-7.42; Fisher's two tailed $\mathrm{p}=0.069)$. After adjusting for sex, the relative odds for disease in those reporting left foot preference were 0.79 (95\% CI $0.18-3.39$; $\mathrm{p}=0.747) 2.59(95 \%$ CI $0.92-7.30 ; \mathrm{p}=0.073)$, and $1.56(95 \%$ CI $0.69-3.56 ; p=0.291)$ for $\mathrm{CD}, \mathrm{UC}$, and IBD, respectively.

Table 2 Relative odds for the association between left handedness and inflammatory bowel diseases (IBD) in two national birth cohorts combined

\begin{tabular}{lclll}
\hline & $\begin{array}{l}\text { No left handed with } \\
\text { disease (\%) }\end{array}$ & $\begin{array}{l}\text { No left handed without } \\
\text { disease (\%) }\end{array}$ & $\begin{array}{l}\text { Unadjusted relative odds } \\
(95 \% \text { CI) }\end{array}$ & $\begin{array}{l}\text { Adjusted relative odds } \\
\text { (95\% CI) }\end{array}$ \\
\hline CD & $8 / 38(21.1 \%)$ & $1919 / 17158(11.2 \%)$ & $2.12(0.97-4.63) \mathrm{p}=0.067$ & $2.12(0.97-4.64) \mathrm{p}=0.059$ \\
UC & $7 / 33(21.2 \%)$ & $1920 / 17163(11.2 \%)$ & $2.14(0.93-4.93) \mathrm{p}=0.089$ & $2.12(0.92-4.90) \mathrm{p}=0.078$ \\
IBD combined & $15 / 71(21 \%)$ & $1912 / 17125(11 \%)$ & $2.13(1.20-3.78) \mathrm{p}=0.008$ & $2.13(1.19-3.77) \mathrm{p}=0.010$ \\
\hline
\end{tabular}

$\mathrm{CD}$, Crohn's disease; UC, ulcerative colitis.

*Adjusted for sex and cohort. 


\section{Discussion}

This study supports the original findings of Geschwind and Behan ${ }^{4}$ and Searleman and Fugagli ${ }^{6}$ by demonstrating a statistically significant twofold increased risk of IBD in left handed subjects. The increased relative odds for foot preference observed for UC also endorses these data.

The study has the advantage of using data from two population based national cohort studies thus avoiding many of the potential biases found in case control studies. Case ascertainment in these studies was likely to be complete as they both reported a very high prevalence of IBD. ${ }^{12}{ }^{13}$ In both cohorts, those responding to the disease surveys at 26 (BCS70) and 33 (NCDS) years were largely representative of the original birth cohorts with some additional loss to follow up of those whose fathers were Registrar General's social class $\mathrm{V}$ at the time of their birth. ${ }^{14}{ }^{15}$ However, social class was not associated with handedness or IBD in either cohort and therefore is unlikely to be a confounding factor. ${ }^{12} 13$

Male subjects were significantly more likely to be left handed than females, a finding that has been previously reported. ${ }^{16}$ However, sex was not associated with the risk of CD, UC, or IBD combined in either cohort in this study, and was therefore unlikely to be a confounding factor as it has been in other studies. Adjusting for sex or cohort did not significantly alter the relationships found between left handedness and IBD (table 2). Cohort members from the BCS70 (born 1970) were more likely to have IBD than those in the NCDS (born 1958) and this follows recent temporal trends in IBD prevalence. ${ }^{12}$

Eleven percent of subjects without IBD reported using their left hand preferentially for writing. This was similar in both cohorts, and is similar to that reported in the control groups of earlier case control studies. ${ }^{6}$ We did not assess the degree of handedness but as we excluded those who responded "either" or "mixed right and left" to handedness questions, subjects included in the study will be those with the most extreme lateralisation of hand preference. Some misclassification of hand and foot preference will have occurred but this is not likely to have resulted in systematic bias as lateralisation was determined at least two years (and usually more than 10 years) before the onset of symptoms of IBD was made in any of the subjects.

Left foot preference was increased in those with UC (OR 2.59) although this was not statistically significant and was based on a small number of cases from one cohort only. However, foot preference is thought to have a less consistent association with cerebral lateralisation than left handedness. ${ }^{17}$

The association between left handedness and IBD is important as it suggests that the two may share common aetiological influences or may indicate a marker of susceptibility. These could reflect shared genetic traits, environmental factors acting in early life, or a combination of these.
One controversial hypothesis suggests that the influences of testosterone (excess production or increased sensitivity to it) in utero may alter growth of the left cerebral hemisphere and the thymus simultaneously, resulting in an association between left handedness and certain $\mathrm{T}$ cell dependent immune disorders. ${ }^{18}$ Some small immunological studies have supported this theory by finding different $\mathrm{T}$ cell subsets, ${ }^{19}$ cytokines, ${ }^{20}$ and autoantibodies ${ }^{21}$ between left and right handers. However, the aetiological processes involved in IBD and other immune mediated diseases reported in association with left handedness are poorly understood and the model has received much criticism. $^{8}$

Seasonal differences in the birth of left handed girls (but not boys) have been reported, ${ }^{22}$ suggesting that environmental factors (possibly infectious agents) may be important. Seasonal differences in birth of subjects with IBD are also described. ${ }^{23}{ }^{24}$ Excess female subjects with IBD, especially CD, have also been reported in some studies. ${ }^{25}$

Genetic explanations of the association between left handedness and autoimmune diseases have been suggested. Different HLA haplotypes have been described between left and right handers that may also be found in some diseases, including $\mathrm{UC},{ }^{26}$ although these are inconsistent. ${ }^{27}$

In summary, this study has found an increased risk of IBD, in particular UC, in left handed subjects from two national birth cohorts. Such an association may help elucidate the aetiological processes leading to IBD although at present the mechanisms remain uncertain.

We are grateful for the assistance of Peter Shepherd, Centre for Longitudinal Studies, Institute of Education, University of London. The Wellcome Trust, Hayward Foundation, Enid Linder Trust, and Crohn's and Colitis in Childhood supported this work.

1 Dellatolas G, Annesi I, Jallon P, et al. An epidemiological reconsideration of the Geschwind-Galaburda theory of reconsideration of the Geschwind-Galaburda theory

2 Smith J. Left-handedness: its association with allergic disease. Neuropsychologia 1987;25:665-74.

3 Soper HV, Satz P, Orsini DL, et al. Handedness patterns in autism suggest subtypes. F Autism Dev Disord 1986;16:15567.

4 Geschwind N, Behan P. Left-handedness: Association with mmune disease, migraine, and developmental learning disorder. Proc Natl Acad Sci USA 1982;79:5097-100.

5 Becker JT, Bass SM, Dew M, et al. Hand preference, immune system disorder and cognitive function among gay/bisexual men: the Multicenter AIDS Cohort Study (MACS). Neuropsychologia 1992;30:229-35.

6 Searleman A, Fugagli AK. Suspected autoimmune disorders and left-handedness: evidence from individuals with diabetes, Crohn's disease and ulcerative colitis. Neuropsydiabetes, Crohn's disease

7 Meyers S, Janowitz HD. Left-handedness and inflammatory Meyers S, Janowitz HD. Left-handedness and in

8 Bryden MP, McManus IC, Bulman-Fleming M. Evaluating Bryden MP, McManus IC, Bulman-Fleming M. Evaluating
the empirical support for the Geschwind-BehanGalaburda model of cerebral lateralisation. Brain Cogn 1994;26:103-67.

9 Mckeever WF, Rich DA. Left handedness and immune disorders. Cortex 1990;26:33-40.

10 Obrzut JE. The Geschwind-Behan-Galaburda theory of cerebral lateralization: thesis, antithesis, and synthesis. Brain Cogn 1994;26:267-74.

11 Ekinsmyth C, Bynner JM, Montgomery SM, et al. An integrated approach to the design and analysis of the 1970 British Cohort Study (BCS70) and the National Child Developish Cohort Study (BCS70) and the National Child Develop-
ment Study (NCDS). London, SSRU, The City University, 1997.

12 Montgomery SM, Morris DL, Thompson NP, et al. Prevalence of inflammatory bowel disease in British 26 year-olds: national longitudinal birth cohort. BMF 1998;316:1058-9. 
13 Thompson NP, Montgomery SM, Pounder RE, et al. Is Thompson NP, Montgomery SM, Pounder RE, et al. Is measles vaccination a risk factor
disease? Lancet 1995;345:1071-4.

14 Shepherd P. Analysis of response bias. In: Ferri E, ed. Life at 33: The fifth follow-up of the National Child Development Study. National Children's Bureau, 1993;184-7.

15 Shepherd P. Survey and response. In: Bynner JM, Ferri E Shepherd P, eds. Twenty-something in the 1990's. UK Ashgate, 1997:129-36.

16 Le Roux A. Sex differences and the incidence of left-handedness. F Psychol 1979;102:261-2.

17 Peter M, Durding BM. Footedness of left- and righthanders. Am f Psychol 1979;92:133-42.

18 Geschwind N, Behan P. Laterality, hormones and immunity. In: Geschwind N, Galaburda AM, eds Cerebral dominance: the biological foundations. Cambridge, Mass:- Harvard University Press, 1984.

19 Yokoyama MM, Hara A, Shiotsuki. Lymphocyte subsets of left-handers. Brain Behav Immun 1987;1:36-9.

20 Chengappa KN, Ganguli R, Yang Z, et al. Non-right sidedness: an association with lower IL-2 production. Life Sci 1992;51:1843-9.
21 Chengappa KN, Ganguli R, Ulrich R, et al. The prevalence of autoantibodies among right and left handed schizo-
phrenic patients and control subjects. Biol Psychiatry 1992; 32:803-11.

22 Leviton A, Kilty T. Seasonal variation in the birth of left-handed schoolgirls. Arch Neurol 1979;36:115-16.

23 Haslam N, Mayberry JF, Probert CS. Month-of-birth is a risk factor for Crohn's disease. Gut 1997;41 (Suppl 3):A175.

24 Morris DL, Montgomery SM, Kyle J, et al. Is season of birth a risk factor for Crohn's disease. Gut 1999;45 (Suppl V):A124.

25 Sandler RS. Epidemiology of inflammatory bowel disease. In: Targan SR, Shanahan F, eds. Inflammatory bowel disease, from bench to bedside. Baltimore: Williams and Wilkins, 1994:5-30.

26 Yeo RA, Gangestad SW. Developmental origins of variation in human hand preference. Genetica 1993;89:281-96.

27 St Marseille A, Braun CMJ. Comments on immune aspects of the Geschwind-Behan-Galburda model and of the article of Bryden, Mcmanus and Bulman-Fleming. Brain Cogn 1994;26:281-90.

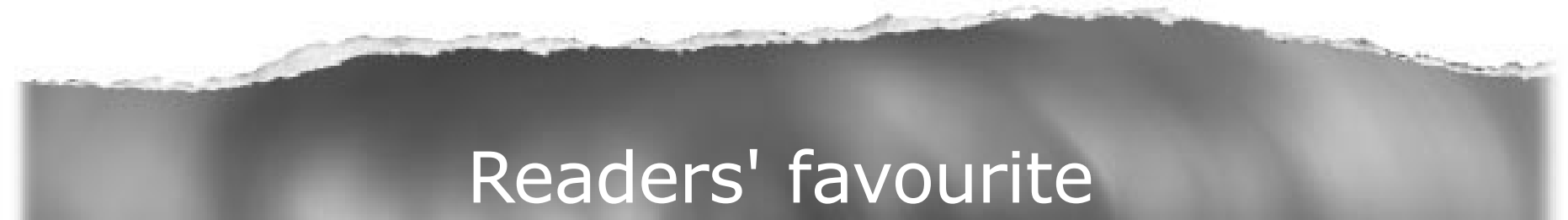

Top 10

Click on the "Top 10" button on the homepage to see which are the best read articles each month

www.gutjnl.com 